then for $t>0$

$$
|w(t)| \leq \frac{\left|a_{n}\right| t^{m-n-1}}{(m-n-1) !}+\frac{\left|a_{n-1}\right| t^{m-n}}{(m-n) !}+\cdots+\frac{\left|a_{0}\right| t^{m-1}}{(m-1) !}
$$

If we replace the restriction that $m \geq 2 n$ by the condition that $w(t) \geq 0$, a similar inequality results and it has a very simple proof.

More specifically, if $w(t)$ is a real-valued non-negative function of the real variable $t$ and is zero for $t<0$ and if its Laplace transform is given by (1) where $m>n$ and the roots of the polynomial $D(s)$ have non-positive real parts, then for $t>0$

$$
w(t) \leq \frac{a_{n} t^{m-n-1}}{(m-n-1) !}+\frac{a_{n-1} t^{m-n}}{(m-n) !}+\cdots+\frac{a_{0} t^{m-1}}{(m-1) !} .
$$

To establish this result, let

$$
F(s)=\frac{a_{n} s^{n}+\cdots+a_{0}}{s^{m}+\cdots+b_{0}} \cdot \frac{s^{m}+\cdots+b_{0}}{s^{m}} .
$$

The corresponding inverse Laplace transform that is zero for $t<0$ may be written as follows for $t>0$.

$$
\begin{aligned}
f(t)= & \frac{a_{n} t^{m-n-1}}{(m-n-1) !}+\cdots+\frac{a_{0} t^{m-1}}{(m-1) !} \\
= & w(t)+b_{m-1} \int_{0}^{t} w(x) d x+\cdots \\
& \quad+\frac{b_{0}}{(m-1) !} \int_{0}^{t}(t-x)^{m-1} w(x) d x .
\end{aligned}
$$

Since the roots of $D(s)$ are real or appear in complex-conjugate pairs and their real parts are all non-positive, every $b_{i}$ is non-negative. Thus, all terms on the right-hand side of (4) are non-negative so that (4) implies (3).

\title{
REFERENCE
}

1. A. H. Zemanian, Further properties of certain classes of transfer functions: II, Quart. Appl. Math. $19,158-159$ (1961)

\section{AN APPLICATION OF THE EULER-MACLAURIN SUM FORMULA TO OPERATIONAL MATHEMATICS*}

By IRVING FRANK (Avco-Everett Research Laboratory, Everett, Mass.)

Introduction. The use of operational methods is especially well adapted to the solution of various problems in applied mathematics. Thus, in problems on heat conduction one is often enabled to find special solutions of the heat balance equation suitable for large or small values of time. It is shown in the present study that the Euler-Maclaurin sum formula may be used in some cases to generate approximate solutions to the heat balance equation which give good results for all values of time, using only a limited number of terms.

${ }^{*}$ Received July 24, 1961. 
Analysis. One form of the Euler-Maclaurin sum formula is [1]

$$
\begin{aligned}
\sum_{k=0}^{\infty} f_{k}=\frac{1}{h} \int_{\alpha}^{\infty} f(x) d x+\frac{1}{2} f(0)-\frac{h}{12} f^{\prime}(0)+\frac{h^{3}}{720} f^{\prime \prime \prime}(0) & -\frac{h^{5}}{30,240} f^{\prime}(0) \\
& +\cdots-\frac{B_{2 m}}{(2 m) !} h^{2 m-1} f_{(0)}^{2 m-1}+E_{m}
\end{aligned}
$$

where $f_{k}=f(a+k h), E_{m}$ is the error term, and

$$
B_{2 m}=\frac{2(-1)^{m}(2 m) !}{(2 \pi)^{2 m}}\left(1+\frac{1}{2^{2 m}}+\frac{1}{3^{2 m}}+\cdots\right)
$$

are the Bernoulli numbers.

In order to illustrate the application of Eq. (1), consider the following problem: the slab $0<x<x_{0}$ with zero initial temperature, insulated at the face $x=0$, while there is a constant heat flux $F$ into the slab at $x=x_{0}$. The Laplace Transform of the temperature at $x=x_{0}$ is given by

$$
L\left[T\left(x_{0}\right)\right]=\frac{F\left(1+\exp \left[-2 q x_{0}\right]\right)}{k p q\left(1-\exp \left[-2 q x_{0}\right]\right)},
$$

where $T$ denotes the temperature, $p$ the Laplace Transform variable, $\alpha$ the diffusivity, $k$ the conductivity, and $q=(p / \alpha)^{1 / 2}$.

Expanding Eq. (3) in negative exponentials, one finds

$$
L\left[T\left(x_{0}\right)\right]=\frac{2 F}{k p q}\left[\frac{1}{2}+\sum_{n=1}^{\infty} \exp \left(-2 n q x_{0}\right)\right] .
$$

It will be noted that the summation in Equation (4) is of the form $\sum_{k=0}^{\infty} f(\alpha+k h)$, where $\alpha=h=2 q x_{0}$.

It will be noted, that $L\left[T\left(x_{0}\right)\right]$ could have been represented as

$$
L\left[T\left(x_{0}\right)\right]=\frac{2 F}{k p q}\left[\sum_{n=0}^{\infty} \exp \left(-2 n q x_{0}\right)-1 / 2\right],
$$

but, as will be shown later, it is preferable to exclude the first term of the expansion from the summation.

Applying Eq. (1) to the term inside the brackets in Eq. (4), we obtain

$$
\begin{aligned}
& L\left[T\left(x_{0}\right)\right]=\frac{F}{k p q}\left\{\frac{1}{2}+\frac{1}{2 q x_{0}} \int_{2 q x_{0}}^{\infty} e^{-\xi} d \xi+e^{-2 q x_{0}}\right. \\
& \cdot\left[\frac{1}{2}+\frac{q x_{0}}{6}-\frac{q^{3} x_{0}^{3}}{90}+\frac{q^{5} x_{0}^{5}}{945}+\frac{B_{2 m}\left(2 q x_{0}\right)^{2 m-1}}{(2 m) !}+\cdots\right],
\end{aligned}
$$

where $f^{n}(0)=-e^{-2 q x_{0}}$ for all $n$.

The inverse transform of Eq. (6) gives

$$
T\left(x_{0}\right)=\frac{F x_{0}}{k}\left[\frac{2}{(\pi)^{1 / 2}} \omega+4 \omega^{2} i^{2} \operatorname{erfc} \frac{1}{\omega}+2 \omega i^{\prime} \operatorname{erfc} \frac{1}{\omega}+\frac{1}{3} \operatorname{erfc} \frac{1}{\omega}-\frac{1}{45} \omega^{3} e^{-\omega^{2}}+\cdots\right],
$$

where

$$
\omega=\left(\frac{\alpha t}{x_{0}^{2}}\right)^{1 / 2}
$$


The solutions suitable for long and short times respectively are [2]

$$
\begin{gathered}
T\left(x_{0}\right)=\frac{F x_{0}}{k}\left[\omega^{2}+1 / 3-\frac{2}{\pi^{2}} \sum_{n=1}^{\infty} \frac{1}{n^{2}} e^{-n^{2} \pi^{2} \omega^{2}}\right], \\
T\left(x_{0}\right)=\frac{F x_{0}}{k}\left[\frac{2}{(\pi)^{1 / 2}} \omega+4 \omega^{2} \sum_{n=1}^{\infty} i^{\prime} \operatorname{erfc} \frac{n}{\omega}\right] .
\end{gathered}
$$

The following table compares $T\left(x_{0}\right)$ as calculated from the first four terms of Eq. (7) with the actual solution accurate to five significant figures for a range of values of $1 / \omega^{2}$.

TABLE I

\begin{tabular}{ccc}
$1 / \omega^{2}$ & $k / F x_{0} T\left(x_{0}\right)$ Eq. (7) & $k / F x_{0} T\left(x_{0}\right)$ Eq. (8) or $(9)$ \\
\hline 0.1 & 10.333 & 10.333 \\
0.2 & 5.3343 & 5.3333 \\
0.5 & 2.3362 & 2.3333 \\
1 & 1.3381 & 1.3333 \\
2.0 & .83580 & .83188 \\
5.0 & .50546 & .50516 \\
10.0 & .35683 & .35684 \\
100.0 & .11284 & .11284 \\
\hline
\end{tabular}

It will be noted that the largest percentage error occurs in the intermediate range of values of $1 / \omega^{2}$, while the approximation to $T\left(x_{0}\right)$ is more accurate for both large and small values of $1 / \omega^{2}$. This occurs because for small values of $1 / \omega^{2}$, or large values of time, the approximation

$$
\sum_{k=0}^{\infty} f(\alpha+k h)=\frac{1}{h} \int_{\alpha}^{\infty} f(x) d x+1 / 2 f(\alpha)
$$

is fairly accurate since $h$ is small and $f(\infty)=0$. On the other hand, for large values of $1 / \omega^{2}$, or small values of time, only the first term of the expansion is really significant, since both $\sum_{k=0}^{\infty} f(\alpha+k h)$ and $1 / h \int_{\alpha}^{\infty} f(x) d x$ are small.

It is evident that the method described here can be used in other applications, where the solution can be represented as $\sum_{n=0}^{\infty} f(\alpha+k h)$ though the accuracy of the approximation will vary from case to case, depending on the function.

\section{REFERENCES}

1. F. B. Hildebrand, Introduction to numerical analysis, McGraw Hill Publishing Co., New York, 1956 , p. 149

2. H. S. Carlslaw and J. C. Jaeger, Conduction of heat in solids, Second edition, Oxford University Press, London 1959, p. 112. 